\title{
Toxicidad subletal de clorpirifos sobre Octolasion cyaneum (Annelida: Oligochaeta, Lumbricidae)
}

Salvio, C.; P.L. Manetti, N.L. Clemente, M.L. Menone y A.N. López

\begin{abstract}
RESUMEN
El objetivo de este trabajo fue evaluar la supervivencia, biomasa, parámetros reproductivos y cambios morfológicos de Octolasion cyaneum (Annelida: Oligochaeta, Lumbricidae) expuesta a diferentes concentraciones de clorpirifos. La unidad experimental consistió en un recipiente con $750 \mathrm{~g}$ de suelo mezclado con la concentración del insecticida y 10 individuos adultos (rango de peso: 300-600 mg). Los tratamientos fueron: 0, 120, 240, 480 (concentración de aplicación recomendada), 960 y 1440 g de clorpirifos $\mathrm{ha}^{-1}$. El diseño fue completamente aleatorizado con cuatro repeticiones y se llevó a cabo en condiciones controladas $\left(20 \pm 2^{\circ} \mathrm{C}\right.$; $\left.14 \mathrm{~L}: 100\right)$. La biomasa de O. cyaneum difirió entre los tratamientos a los 7,14 y 28 días después de la aplicación (DDA) $(p<0,05)$, observándose la biomasa mayor en el testigo. El número de cocones y juveniles a los 56 DDA fue mayor en el testigo con respecto a los demás tratamientos $(p<0,05)$. Se obtuvieron 235 individuos vivos y 5 muertos, no obstante, el $35 \%$ de los organismos presentaron cambios morfológicos. Se observó que el porcentaje de daño varió en función de la concentración del insecticida $(p=0,0046)$. Clorpirifos no afectó la supervivencia de $O$. cyaneum pero causó cambios morfológicos y reducción en su biomasa y en sus parámetros reproductivos.
\end{abstract}

Palabras clave: lombriz, bioensayo ecotoxicológico, insecticida neurotóxico.

Salvio, C.; P.L. Manetti, N.L. Clemente, M.L. Menone and A.N. López, 2015. Sublethal toxicity of chlorpyrifos on Octolasion cyaneum (Annelida: Oligochaeta, Lumbricidae). Agriscientia 32 (2): 131-138

\section{SUMMARY}

The aim of this study was to evaluate the survival, biomass, reproductive parameters and morphological changes of Octolasion cyaneum (Annelida: Oligochaeta, Lumbricidae) exposed to different concentrations of chlorpyrifos. The experimental unit consisted of a container with $750 \mathrm{~g}$ of soil mixed with the concentration of the insecticide and 10 adult individuals (weight range: 300$600 \mathrm{mg}$ ). The treatments were: 0, 120, 240, 480 (recommended application concentration), 960 and $1440 \mathrm{~g}$ of chlorpyrifos ha-1. The design was completely randomized with four replications and was conducted under controlled 
conditions $\left(20 \pm 2^{\circ} \mathrm{C}, 14 \mathrm{~L}: 100\right)$. O. cyaneum biomass differed between treatments at 7,14 and 28 days after application (DAA) $(p<0.05)$, and showed the highest biomass in the control. The number of cocoons and juveniles at 56 DAA was higher in the control compared to the other treatments $(p<0.05)$. In the bioassay 235 individuals were alive and 5 were dead, nevertheless the $35 \%$ of organisms showed morphological changes. It was noted that the percentage of damage varied depending on the concentration of the insecticide $(p=0.0046)$. Chlorpyrifos did not affect the survival of $O$. cyaneum but caused morphological changes and a reduction in biomass and reproductive parameters.

Key words: earthworm, ecotoxicological bioassay, neurotoxic insecticide.

C. Salvio, P.L. Manetti, N.L. Clemente y A.N. López: Facultad de Ciencias Agrarias, Universidad Nacional de Mar del Plata - Estación Experimental del Instituto Nacional de Tecnología Agropecuaria (INTA). C.C. 276 (7620) Balcarce, Argentina. M.L. Menone. Laboratorio de Ecotoxicología. IIMYC CONICET/ UNMdP. Funes 3350 (7600), Mar del Plata. Argentina. Correspondencia a: salvio.carla@inta.gob.ar.

\section{INTRODUCCIÓN}

Las lombrices son frecuentes en una amplia gama de suelos y entre los invertebrados son los más abundantes, representando un $92 \%$ de la biomasa del suelo (Wu et al., 2011). Son considerados "ingenieros del ecosistema" debido a que realizan galerías formando bioporos que contribuyen notablemente en aumentar la infiltración del agua y la aireación del suelo, mejorando su drenaje y evitando su compactación (Sanchez-Hernandez, 2006). Mediante estas funciones, colaboran con el mantenimiento de la estructura y fertilidad del suelo, participan en la descomposición de la materia orgánica y en el ciclado de los nutrientes, estimulando el crecimiento de las plantas y favoreciendo la heterogeneidad del hábitat (Paoletti, 1999). De esta manera, estos organismos participan en los servicios ecosistémicos del suelo y son considerados potenciales indicadores de la salud de éste (Lavelle et al., 2006).

La aplicación intensiva de los productos fitosanitarios en el mundo, según el tipo de ingrediente activo (i.a.) y su concentración, puede afectar a organismos no blanco y, en el largo plazo, conducir al desarrollo de daños irreversibles tanto en la estructura como en la función del ecosistema suelo (Reinecke \& Reinecke, 2007). Clorpirifos es uno de los insecticidas organofosforados más utilizados tanto en la Argentina como en otras partes del mundo, ya que posee un amplio espectro de acción sobre los artrópodos plagas (Micucci \& Taboada, 2006). Este neurotóxico actúa sobre el sistema nervioso central inhibiendo la acción de la acetilcolinestera- sa (AChE), enzima que regula la actividad biológica del neurotransmisor acetilcolina (ACh) (Corbett et al., 1984). Debido a su uso generalizado los invertebrados benéficos edáficos, como las lombrices, se encuentran expuestos a la acción tóxica de este insecticida. Se sabe que los plaguicidas pueden afectar a las lombrices ya sea por contacto dermal, cuando los tóxicos atraviesan el tegumento alcanzando el líquido celómico y se transportan por el cuerpo, o por ingestión cuando se alimentan de los residuos vegetales contaminados (SanchezHernandez, 2006). La mayoría de los estudios sugieren que el tegumento constituye una de las vías de ingreso de las sustancias tóxicas que producen cambios morfológicos sobre esos organismos (Jager et al., 2003; Vijver et al., 2005). Además, varios autores han encontrado que la aplicación de clorpirifos disminuyó tanto la biomasa como los parámetros reproductivos de las lombrices expuestas (Booth \& O'Halloran, 2001; lannacone \& Alvariño, 2004; Zhou et al., 2007; De Silva et al., 2010).

Por otra parte, las lombrices poseen quimiorreceptores en la superficie del cuerpo que les proporcionan una sensibilidad elevada a los químicos que se encuentran en el suelo (Reinecke \& Reinecke, 2007) y, en consecuencia, son considerados como biondicadores de los factores antropogénicos de estrés (Römke et al., 2005). Eisenia fetida es la especie más utilizada en los bioensayos de ecotoxicidad debido a que posee un ciclo de vida corto, fecundidad y sensibilidad elevada a los químicos (Lowe \& Butt, 2007; Momo \& Falco, 2010). Sin embargo, como esta especie es epígea (se ubica por encima del suelo) su desventaja princi- 
pal es que no se encuentra habitualmente presente en los suelos cultivados, por lo cual se debería tener en cuenta otra especie de lombriz como bioindicador. En este sentido, Octolasion cyaneum es abundante y representativa de los suelos del sudeste bonaerense (Salvio, 2012). Se trata de una especie endogeíca y su valor indicativo es medioalto; es sensible a ambientes con niveles bajos de materia orgánica y a las perturbaciones causadas por los sistemas de labranza convencional (Butt, 1993; Momo \& Falco, 2010).

El objetivo de este trabajo fue evaluar la supervivencia, la biomasa, los parámetros reproductivos y los cambios morfológicos de $O$. cyaneum expuesta a diferentes concentraciones de clorpirifos.

\section{MATERIALES Y MÉTODOS}

Se utilizaron ejemplares adultos clitelados de $O$. cyaneum, los cuales fueron extraídos manualmente en un lote bajo siembra directa ubicado en la Estación Experimental Agropecuaria (EEA) del Instituto Nacional de Tecnología Agropecuaria (INTA) Balcarce, Argentina ( $37^{\circ} 45^{\prime} \mathrm{S} ; 58^{\circ} 18^{\prime} \mathrm{O}, 120 \mathrm{~m}$ s.n.m.). Estos ejemplares fueron colocados en recipientes de plástico de $36,5 \mathrm{~cm} \times 24,5 \mathrm{~cm}$ con 10 $\mathrm{cm}$ de suelo húmedo y se llevaron a una cámara de cría a $20 \pm 2^{\circ} \mathrm{C}$ y $14 \mathrm{~L}: 100$ para su aclimatación durante dos semanas.

Para el bioensayo, la unidad experimental consistió en un recipiente de plástico de $11,5 \mathrm{~cm}$ de diámetro y $11 \mathrm{~cm}$ de altura, provisto de una tapa perforada, en el cual se colocaron $750 \mathrm{~g}$ de suelo mezclado con la concentración del insecticida y 10 individuos adultos clitelados con un rango de peso de 300-600 mg (OECD, 1984, 2004). Los tratamientos fueron: 0, 120, 240, 480 (concentración de aplicación recomendada), 960 y $1440 \mathrm{~g}$ de i.a. de clorpirifos ha-1 $(48 \%$ de i.a., concentrado emulsionable: CE, marca comercial Lorsban $48 \mathrm{EC} \otimes$ ). Para lograr cada una de las concentraciones de i.a. por kg de suelo seco, se realizaron diluciones del insecticida en agua destilada, teniendo en cuenta que el peso de suelo de una hectárea a $5 \mathrm{~cm}$ y con una densidad aparente de $1,2 \mathrm{Mg} \mathrm{m}^{-3}$ equivale a $600.000 \mathrm{~kg}$. Las aplicaciones del insecticida disuelto en agua se realizaron con un pulverizador manual sobre una capa fina y uniforme de suelo de $5 \mathrm{~cm}$ de altura sobre un paño de nylon de 50 $\mathrm{cm}$ por $50 \mathrm{~cm}$ de lado. Luego, para lograr una incorporación adecuada del producto al sustrato, se realizaron 20 movimientos de las partes laterales del paño hacia el centro. Las propiedades físicas y químicas del suelo utilizado fueron las siguientes: $5,1 \%$ de materia orgánica; $20,9 \%$ de arcilla; $34,9 \%$ de limo; 44,2\% de arena; $\mathrm{pH}=7,7$; conductividad $0,6 \mathrm{mmhos} \mathrm{cm}^{-1}$ y capacidad de intercambio catiónica (C.I.C.) 29,3 meq $100 \mathrm{~g}^{-1}$.

El diseño fue completamente aleatorizado con cuatro repeticiones y los recipientes se colocaron en una cámara a $20 \pm 2^{\circ} \mathrm{C}$ y $14 \mathrm{~L}: 100$. Se midieron las siguientes variables: el número de individuos vivos y/o muertos, la biomasa en peso húmedo (mg) y los cambios morfológicos a los 7, 14, 21 y 28 días después de la aplicación (DDA); además, se contaron el número de cocones y de juveniles a los 56 DDA. En cada observación se controló la humedad del suelo y cuando fue necesario se pulverizó el sustrato con agua destilada. La biomasa de los ejemplares se registró mediante una balanza analítica electrónica (precisión = 0,001 g). Los cambios morfológicos observados se clasificaron en las siguientes categorías: 1) sin daño; 2) daño leve (cambio de color en la totalidad del cuerpo del organismo); 3) daño moderado (decoloración en los segmentos posteriores del cuerpo); 4) daño severo (desprendimiento de la parte posterior del cuerpo); 5) daño total (muerte del organismo). Se determinó el porcentaje de daño ponderado (\%) en función de las concentraciones del insecticida de acuerdo a la fórmula de Townsend \& Herberger (1943) adaptada:

$$
\mathrm{PD}(\%)=\left[\Sigma\left(\mathrm{n}^{*} \mathrm{v}\right) / \mathrm{CM}^{*} \mathrm{~N}\right]^{*} 100
$$

donde PD = promedio ponderado de daño (\%), $\mathrm{n}=$ número de organismos por cada categoría; $\mathrm{v}=$ valor numérico de cada categoría; $\mathrm{CM}=$ valor de la categoría mayor y $\mathrm{N}$ = número total de organismos por tratamiento. Se analizó el PD (\%) en función de cada concentración del plaguicida a los 28 DDA.

Las variables se analizaron mediante el análisis de la varianza (ANOVA), previa validación del supuesto de homogeneidad de varianzas mediante la prueba de Levene $(\alpha=0,05)$. Cuando se detectaron diferencias entre las medias se realizó la prueba de mínimas diferencias significativas (MDS) ( $\alpha$ $=0,05)$. Todos los análisis se realizaron con el programa R 3.1.1 (R Development Core Team, 2014).

\section{RESULTADOS Y DISCUSIÓN}

En la biomasa de $O$. cyaneum se observaron diferencias significativas entre los distintos tratamientos a los $7(p=0,0001), 14(p=0,01)$ y 28 DDA $(p=0,04)$. Tanto a los 7 como a los 28 DDA, la biomasa fue mayor en el testigo con respecto a los restantes tratamientos (Figura 1). A los 7 DDA se observó una disminución de $95 \mathrm{mg}$ en el peso de las lombrices entre el testigo y el tratamiento 


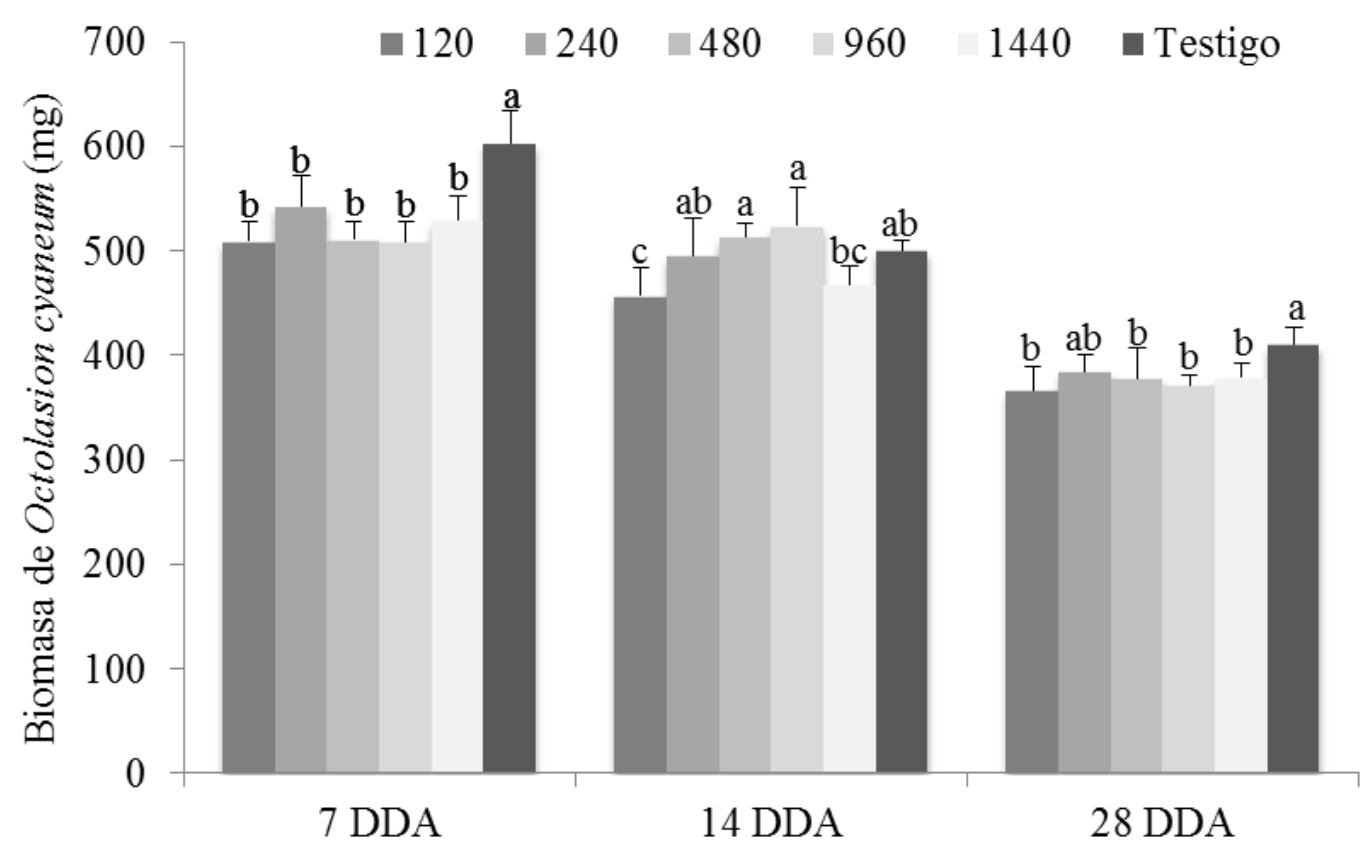

Figura 1. Biomasa de Octolasion cyaneum (mg) (media \pm desvío estándar) en los diferentes tratamientos con clorpirifos ( $\mathrm{g}$ de i.a. ha-1) a los 7, 14 y 28 días después de la aplicación (DDA). Barras con las letras iguales en cada fecha de observación indican diferencias no significativas entre los tratamientos (MDS, $p>0,05)$.

con el peso menor obtenido, mientras que en las siguientes fechas de observación la disminución se mantuvo constante en $43 \mathrm{mg}$. Además, en todos los tiempos de observación se obtuvo un peso menor de los individuos con la concentración menor de clorpirifos con respecto al testigo (Figura 1).

Por otro lado, los parámetros reproductivos en O. cyaneum como el número de cocones y de juveniles difirieron entre los tratamientos $(p<0,05)$. El número de cocones fue mayor en el testigo con respecto a los demás tratamientos a los 56 DDA ( $p$ $=0,001$ ), obteniéndose 15 cocones en el testigo y 10 cocones con $120 \mathrm{~g}$ de clorpirifos ha ${ }^{-1}$ (Figura 2). En cuanto al número de juveniles se observó la misma tendencia que con los cocones, observándose el número mayor en el testigo y el menor con las concentraciones menores, 120 y 240 g i.a. ha-1 (Figura 2).

De manera similar a los resultados obtenidos en este estudio, Booth et al. (2000) obtuvieron una disminución en el peso de Aporrectodea caliginosa así como una reducción en la producción de cocones con 0,004 y 0,028 $\mathrm{g}$ de clorpirifos $\mathrm{kg}^{-1}$ de suelo. En coincidencia, Zhou et al. (2007) observaron similares efectos sobre $E$. fetida con dosis comprendidas entre 0,005 y 0,080 $\mathrm{g}$ de clorpirifos $\mathrm{kg}^{-1} \mathrm{de}$ suelo. Por otro lado, lannacone \& Alvariño (2004) detectaron que clorpirifos no afectó la superviven- cia de E. fetida, pero en cambio produjo una disminución en el peso de los organismos. También, De Silva et al. (2010) determinaron una reducción en el número de adultos y de juveniles con 4,4 y 8,8 $\mathrm{kg}$ de clorpirifos ha-1 después de los tres meses de su aplicación; asimismo, obtuvieron una reducción en la biomasa, la abundancia y la tasa de descomposición de los residuos. De manera similar, Alshawish et al. (2004) observaron un impacto significativo en la fecundidad de $A$. caliginosa con 0,050 $\mathrm{g}$ de clorpirifos $\mathrm{kg}^{-1}$ de suelo. Resultados similares informaron Zhou et al. (2011), quienes obtuvieron una disminución en el número de cocones y de juveniles de E. fetida después de ocho semanas de exposición. A su vez, Rafael (2014) observó que la biomasa de $O$. cyaneum difirió a los 14 DDA y disminuyó con una concentración cinco veces mayor que la concentración habitual de aplicación en el campo.

Con las concentraciones diferentes de clorpirifos se obtuvieron 235 individuos vivos de 0 . cyaneum y 5 muertos. No obstante, el 35\% de los organismos expuestos a las diferentes concentraciones de clorpirifos presentaron cambios morfológicos. A los 28 DDA, el porcentaje de daño (PD) difirió entre los tratamientos con respecto al testigo $(p=0,004)$. Con $240 \mathrm{~g}$ de i.a. y $1440 \mathrm{~g}$ de i.a. se observaron los daños mayores, siendo sus valores 


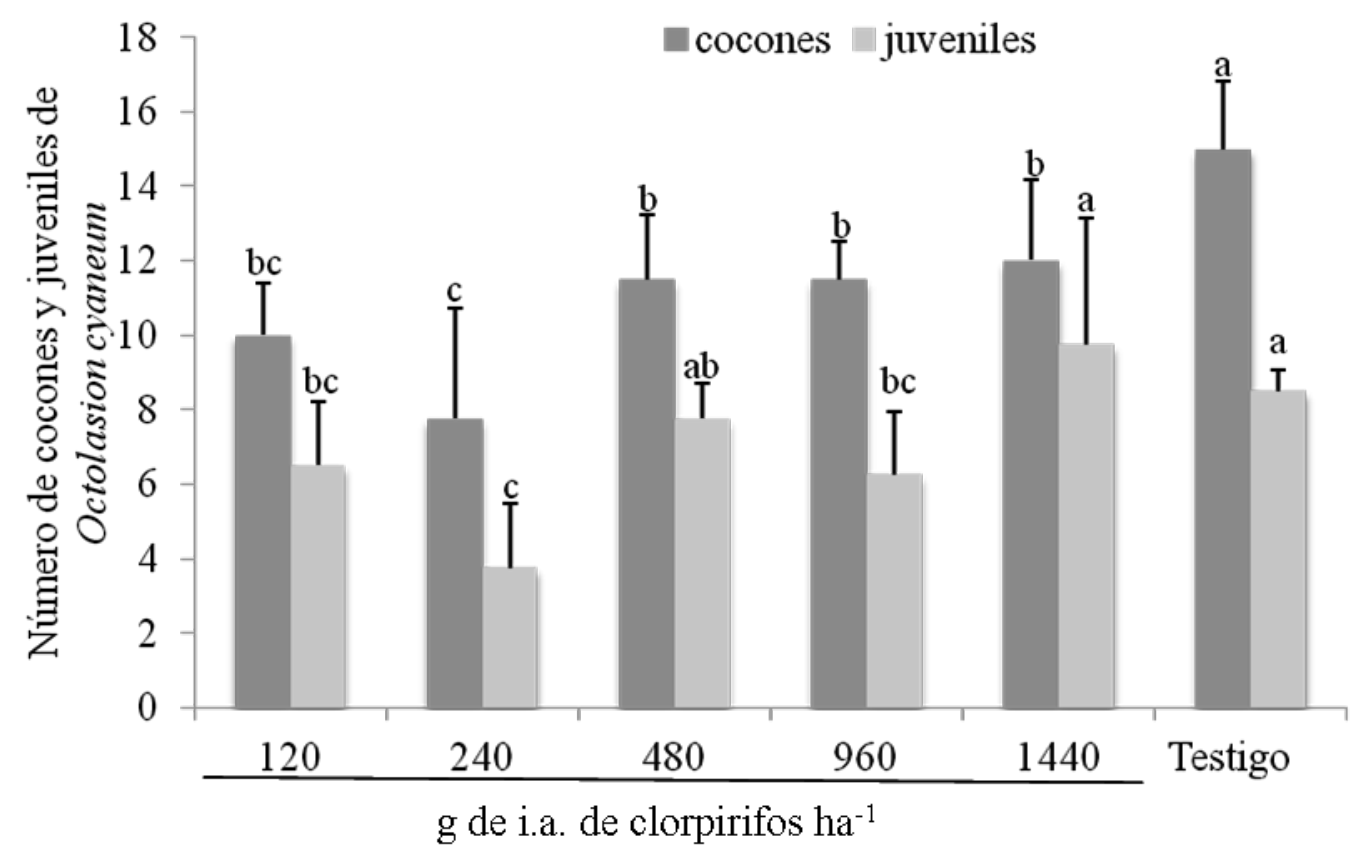

Figura 2. Número de cocones y juveniles de Octolasion cyaneum (media \pm desvío estándar) en los diferentes tratamientos con clorpirifos ( $\mathrm{g}$ de i.a. ha-1) a los 56 días después de la aplicación. Barras con las letras iguales indican diferencias no significativas entre los tratamientos (MDS, $p>0,05$ ).

Tabla 1. Daño de Octolasion cyaneum (\%) (media \pm desvío estándar) en las diferentes concentraciones de clorpirifos (g i.a. ha-1) a los $28 \mathrm{DDA}^{(1)}$.

\begin{tabular}{cc}
\hline $\begin{array}{c}\text { Concentraciones de clorpirifos } \\
(\mathrm{g} \mathrm{i.a.} \mathrm{ha-1)}\end{array}$ & Daño (\%) \\
\hline 120 & $10,62 \pm 6,65 \mathrm{ab}$ \\
240 & $18,13 \pm 7,81 \mathrm{a}$ \\
480 & $2,20 \pm 3,06 \mathrm{c}$ \\
960 & $9,06 \pm 5,34 \mathrm{ab}$ \\
1440 & $15,00 \pm 9,63 \mathrm{a}$ \\
\hline
\end{tabular}

(1) Letras iguales indican diferencias no significativas entre los tratamientos (MDS, $p>0,05)$.

de 18 y $15 \%$ respectivamente $(p<0,05)$ (Tabla 1$)$. Mientras que, con la concentración de aplicación recomendada $480 \mathrm{~g}$ de i.a. ha- ${ }^{-1}$, se obtuvo un PD menor: 2,5\% (Tabla 1). El PD mayor obtenido con la dosis menor, $240 \mathrm{~g}$ de i.a. ha-1 (Tabla 1), se corresponde con los parámetros reproductivos obtenidos, ya que con esa misma concentración tanto el número de cocones como el de juveniles disminuyeron con respecto al testigo (Figura 2). Por otro parte, el daño leve tuvo un predominio mayor con respecto a los daños moderado y severo. Además, en la minoría de los individuos se produjo un abultamiento cercano a la región clitelar y lesiones en el tegumento, como así también enrollamiento, ondulaciones del cuerpo y producción excesiva de mucus que provocaron lentitud en sus movimientos (Figura 3). De la misma manera, Rafael (2014) observó que $33 \%$ de los organismos de O. cyaneum presentaron cambios morfológicos similares y el PD aumentó a medida que la concentración de clorpirifos se incrementó. Síntomas similares fueron observados en E. fetida expuesta a clorpirifos (Rao et al., 2003), temefos (Hackenberger et al., 2008) y profenofos (Reddy \& Rao, 2008). Además, en este trabajo y en coincidencia con otros autores, un efecto característico del organofosforado fue el desprendimiento de la región posterior del organismo (daño severo), que se explica debido a que el organismo requiere para su recuperación energía que la obtiene de la autólisis producida en dicha región (Figura 3) (Ramaswami \& Subbram, 1992). Asimismo, la utilización de sus propios tejidos puede ser considerada como una ventaja adaptativa para superar las condiciones adversas, debido a que se trata de proteger los órganos vitales del organismo que se encuentran en su mayoría en la región anterior. Por otra parte, clorpirifos al ser un insecticida neurotóxico actúa sobre el sistema nervioso central del organismo inhibiendo la acción de la acetilcolinesterasa (AChe); aunque la inhibición no es perjudicial per se ocasiona acumulación del neurotransmisor acetilcolina (ACh) y causa una estimulación continua de la actividad nerviosa 


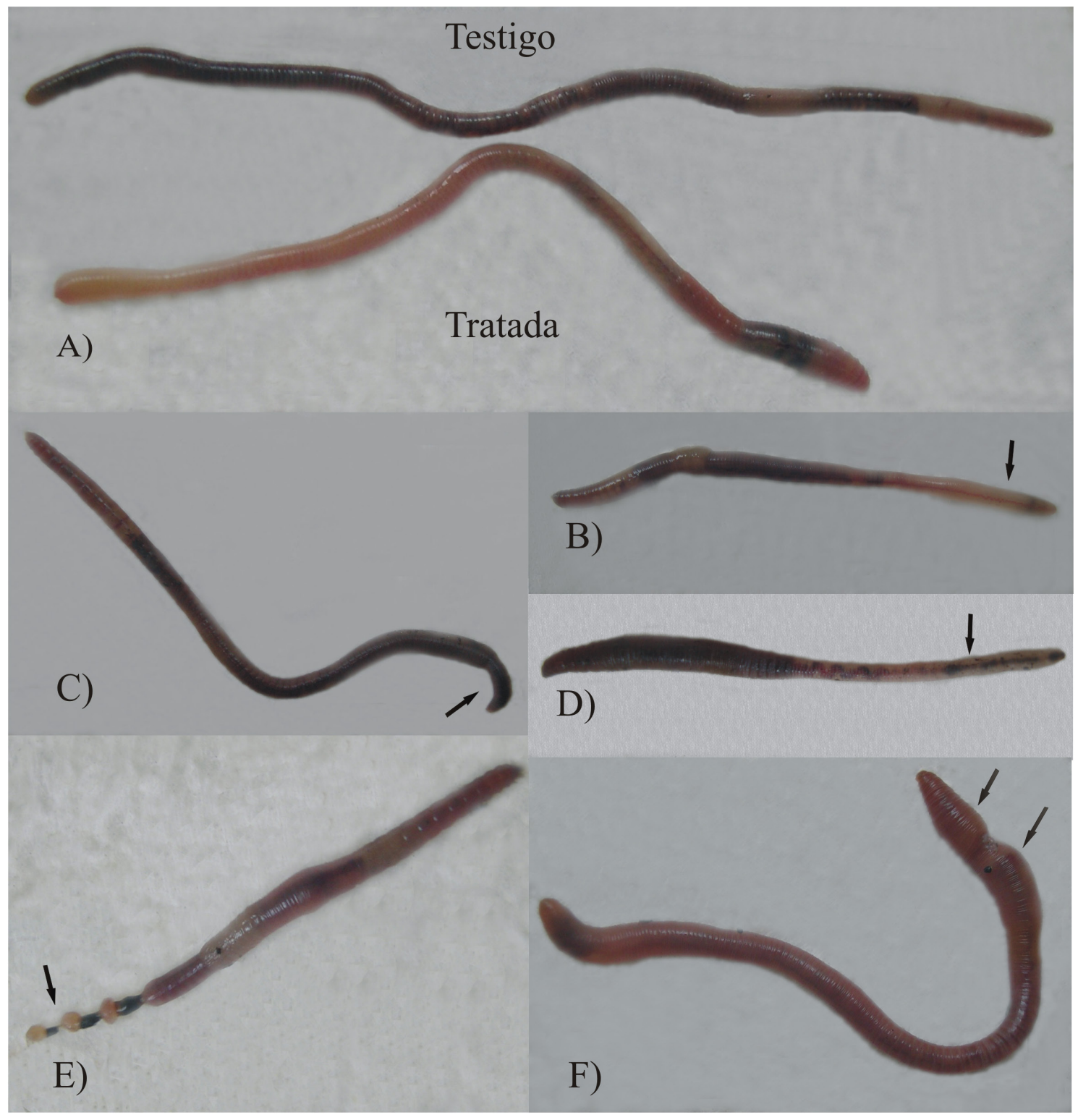

Figura 3. Síntomas de toxicidad de Octolasion cyaneum producto de la exposición a clorpirifos: A) cambio de coloración del organismo tratado en comparación con el testigo, B), C), D) y E) proceso de transición de la decoloración en la parte posterior del cuerpo del organismo y pérdida de dicha región y F) abultamiento cercano a la zona clitelar y lesiones en el tegumento.

(Rao et al., 2003; Reinecke \& Reinecke, 2007). En consecuencia, clorpirifos al inhibir la AChe en las lombrices ocasiona un comportamiento anormal de los organismos que influye en la alimentación y en la reproducción de estos oligoquetos (Rao et al., 2003; Reinecke \& Reinecke, 2007). En coincidencia con el presente estudio, en O. cyaneum se observó dicho comportamiento.

\section{CONCLUSIONES}

Estos estudios corroboran que la concentración habitual de aplicación de $480 \mathrm{~g}$ de clorpirifos ha-1 no afecta la supervivencia de $O$. cyaneum, pero causa efectos subletales en los organismos expuestos, como cambios morfológicos y disminución en la biomasa y en los parámetros reproductivos de la especie. Por lo tanto, se debería seleccionar adecuadamente el principio activo y 
su concentración de aplicación para asegurar la conservación y el equilibrio biológico de los organismos que viven en el sistema suelo.

\section{BIBLIOGRAFÍA}

Alshawish, S.A.; A.I. Mohamed and G.A. Nair, 2004. Prolonged toxicity of sub-lethal dosages of chemical pesticides on the body mass and cocoons of Aporrectodea caliginosa (Savigny 1826) (Oligochaeta: Lumbricidae) inhabiting Benghazi, Libya[J]. Proceendings of the National Academy of Sciences India 74 (B): 123-133.

Booth, L.H.; V.J. Heppelthwaite and K. O'Halloran, 2000. Growth, development and fecundity of the earthworm Aporrectodea caliginosa after exposure to two organophosphates. New Zealand Plant Protection 53: 221225.

Booth, L.H. and K. O'Halloran, 2001. A comparison of biomarker responses in the earthworm Aporrectodea caliginosa to the organophosphorus insecticides diazinon and chlorpyrifos. Environmental Toxicology and Chemistry 20 (11): 2494-2502.

Butt, K.R., 1993. Reproduction and growth of three deepburrowing earthworms (Lumbricidae) in laboratory culture in order to assess production for soil restoration. Biology and Fertility of Soils 16: 135-138.

Corbett, J.R.; K. Wright and A.C. Baillie, 1984. The biochemical mode of action of pesticides. Academic Press. London. England. 382 pp.

De Silva, P.M.C.S.; A. Pathiratne, N.M. Van Straalen and C.A.M. Van Gestel, 2010. Chlorpyrifos causes decreased organic matter decomposition by suppressing earthworm and termite communities in tropical soil. Environmental Pollution 158: 3041-3047.

Hackenberger, B.K.; D. Jari-Perkuš and S. Stepi, 2008. Effect of temephos on cholinesterase activity in the earthworm Eisenia fetida (Oligochaeta, Lumbricidae). Ecotoxicology and Environmental Safety 71: 583-589.

lannacone, J. and L. Alvariño, 2004. Eisenia fetida (Savegny, 1826) (Annelida: Lumbricidae) como modelo ecotoxicológico para evaluar lindano y clorpirifos. Acta Zoológica Lilloana 48 (1-2): 5-12.

Jager, T.; R.H.L.J. Fleuren, E.A. Hogendoorn and G. De Korte, 2003. Elucidating the routes of exposure for organic chemicals in the earthworm, Eisenia andrei (Oligochaeta). Environmental Science and Technology 37: 3399-3404.

Lavelle, P.; T. Decaëns, M. Aubert, S. Barot, M. Blouin, F. Bureau, P. Margarie, P. Mora and J.P. Rossi, 2006. Soil invertebrates and ecosystem services. European Journal of Soil Biology 42: 3-15.

Lowe, C.N. and K.R. Butt, 2007. Earthworm culture, main- tenance and species selection in chronic ecotoxicological studies: A critical review. European Journal of Soil Biology 43: 281-288.

Micucci, F. and M. Taboada, 2006. Soil physical properties and soybean (Glycine max, Merrill) root abundance in conventionally- and zero-tilled soils in the humid Pampas of Argentina. Soil and Tillage Research 86: 152-162.

Momo, F.R. y L.B. Falco, 2010. Las lombrices de tierra. En: Momo, F.R. y L.B. Falco (Eds.), Biología y ecología de la fauna del suelo. Imago Mundi. Argentina. pp. 141-160.

OECD (Organisation for Economic Cooperation and Development), 1984. Guidelines for the testing of chemicals: earthworm acute toxicity tests. Paris, France. 9 $\mathrm{pp}$.

OECD (Organisation for Economic Cooperation and Development), 2004. Guidelines for the testing of chemicals: earthworm reproduction test (Eisenia fetidal Eisenia andrel). Paris, France. 18 pp.

Paoletti, M.G., 1999. The role of earthworms for assessment of sustainability and as bioindicators. Agriculture Ecosystems and Environment 74:137-155.

R Development Core Team, 2014. R: a language and environment for statistical computing. R Foundation for Statistical Computing, Vienna, Austria. Version 3.1.1.

Rafael, S.E., 2014. Comportamiento evasivo de Octolasion cyaneum (Annelida: Oligochaeta, Lumbricidae) y efectos letales y subletales de su exposición a la acción de plaguicidas. Tesis de grado. Facultad de Ciencias Agrarias, Universidad Nacional de Mar del Plata. $61 \mathrm{pp}$.

Ramaswami, V. and V. Subbram, 1992. Effect of selected textile dye on the survival, morphology, and burrowing behavior of the earthworm Polypheretima elongata. Bulletin of Environmental Contamination and Toxicology 48: 253-258.

Rao, J.V.; Y.S. Pavan and S.S. Madhavendra, 2003. Toxic effects of chlorpyrifos on morphology and acetylcholinesterase activity in the earthworm, Eisenia foetida. Ecotoxicology and Environmental Safety 54: 296-301.

Reddy, N.C. and J.V. Rao, 2008. Biological response of earthworm, Eisenia foetida (Savigny) to an organophosphorous pesticide, profenofos. Ecotoxicolology and Environmental Safety 71: 574-582.

Reinecke, S. and A. Reinecke, 2007. Biomarker response and biomass change of earthworms exposed to chlorpyrifos in microcosms. Ecotoxicolology and Environmental Safety 66: 92-101.

Römke, J.; S. Jansch and W. Didden, 2005. The use of earthworms in ecological soil classification and assessment concepts. Ecotoxicolology and Environmental Safety 62: 249-265. 
Salvio, C., 2012. Impacto de las prácticas agrícolas sobre poblaciones de Milax gagates y Armadillidium vulgare e invertebrados benéficos en soja bajo siembra directa. Tesis Magíster Scientiae. Facultad de Ciencias Agrarias, Universidad Nacional de Mar del Plata. 134 pp.

Sanchez-Hernandez, J.C., 2006. Earthworm Biomarkers in Ecological Risk Assessment. Reviews of Environmental Contamination and Toxicology 188: 85-126.

Townsend, G.R. and J.W. Herberger, 1943. Methods for estimating losses caused by diseases in fungicide experiment. Plant Disease Report 34:340-343.

Vijver, M.G.; H.T. Wolterbeek, J.P.M. Vink and C.A.M. Van Gestel, 2005. Surface adsorption of metals onto the earthworm Lumbricus rubellus and the isopod Porce-
Ilio scaber is negligible compared to absorption in the body. Science of the Total Environmental 340: 271-280.

Wu, X.M.; Y.L. Yu, M. Li, Y.H. Long, H. Fang and S.N. Li, 2011. Prediction of bioavailability of chlorpyrifos residues in soil to earthworms. Journal of Soil Science and Plant Nutrition 11(1): 44-57.

Zhou, S.; C. Duan, H. Fu, Y. Chen, X. Wang and Z. Yu, 2007. Toxicity assessment for chlorpyrifos-contaminated soil with three different earthworm test methods. Journal of Environmental Sciences 19: 854-858.

Zhou, S.; C. Duan, W.H. Gi Michelle, F. Yang and X. Wang, 2011. Individual and combined toxic effects of cypermethrin and chlorpyrifos on earthworm. Journal of Environmental Sciences 23(4): 676-680. 